\title{
Electrically charged strange quark stars with a non-linear equation-of-state
}

\author{
Grigoris Panotopoulos ${ }^{1, a}$, Ángel Rincón ${ }^{2, b}$ \\ ${ }^{1}$ Departamento de Física, Centro de Astrofísica e Gravitação, Instituto Superior Técnico-IST, Universidade de Lisboa-UL, Av. Rovisco Pais, \\ 1049-001 Lisbon, Portugal \\ ${ }^{2}$ Instituto de Física, Pontificia Universidad Católica de Valparaíso, Avenida Brasil 2950, Casilla, 4059 Valparaíso, Chile
}

Received: 10 February 2019 / Accepted: 11 June 2019 / Published online: 18 June 2019

(C) The Author(s) 2019

\begin{abstract}
The properties of electrically charged strange quark stars are investigated. We assume a non-linear equation-of-state, and we obtain numerical solutions to the structure equations. The key features of the solutions obtained here are (i) they can support a 2 solar masses star, (ii) both the mass and the electric charge of the stars increase with the $\alpha$ parameter characterizing the electric density, (iii) the electric object is heavier and larger than its neutral counterpart.
\end{abstract}

\section{Introduction}

Compact objects [1-3] are the final fate of stars, and since they are characterized by ultra high matter densities the nonrelativistic Newtonian description is not adequate. Dense, compact objects are relativistic and as such they are described properly within the framework of Einstein's General Relativity (GR) [4]. On the one hand neutron stars were predicted to exist by Baade and Zwicky [5] soon after the discovery of the neutron by James Chadwick [6,7]. Indeed, several decades later the discoveries of pulsars in the Crab and Vela supernova remnants [8] led to their identification as neutron stars one year after the discovery of pulsars in 1967 [9].

On the other hand quark matter, by assumption, is absolutely stable, and it may be the true ground state of hadronic matter $[10,11]$. Therefore a new class of compact objects has been postulated to exist as an alternative to neutron stars. As a matter of fact, they provide us with a plausible explanation of the puzzling observation of some super-luminous supernovae $[12,13]$, which occur in about one out of every 1000 supernovae explosions, and are more than 100 times brighter than normal supernovae. The compact objects called "strange

\footnotetext{
a e-mail: grigorios.panotopoulos@tecnico.ulisboa.pt

be-mail: angel.rincon@pucv.cl
}

quark stars" [14-19], since they are a much more stable configuration compared to neutron stars, may explain the origin of the huge amount of energy released in super-luminous supernovae.

Since real astronomical objects are expected to be electrically neutral, or at least without a significant amount of electric charge, in studies of compact relativistic astrophysical objects the authors usually focus on electrically neutral stars made of an isotropic fluid, and the interior solution is matched to the exterior Schwarzschild solution [20] on the surface of the object. However, after the work of Bekenstein [21] there is nowadays some interest in adding electric charge to matter inside the star. Over the years some people have investigated the properties of electric stars. However, previous studies have considered either incompressible, uniform density stars, or polytropic stars and NSs [22-28]. Regarding strange quark stars with a non-vanishing electric charge, we are aware of the works of $[29,30]$, where the well-studied linear equation-of-state for quark matter was used.

Despite the fact they are still theoretical objects, in the present work we propose to investigate the properties of electrically charged strange quarks stars adopting a non-linear equation-of-state instead, obtained in the framework of color superconductivity [31-33], assuming a non-vanishing energy gap and mass for the $s$ quark. The assumed charge density, too, here is different than the ansatz made in $[29,30]$.

The plan of our work is the following: after this introduction, we present the structure equations describing hydrostatic equilibrium in Sect. 2, while in the third section we show and discuss our numerical results. Finally we conclude our work in the last section. We adopt the mostly positive metric signature, $(-,+,+,+)$, and we work in natural units where the speed of light in vacuum $c$ as well as the reduced Planck constant $\hbar$ are set to unity, $c=1=\hbar$. In these units all dimensionful quantities are measured in $\mathrm{GeV}$, 
and we make use of the following conversion rules $1 \mathrm{~m}=$ $5.068 \times 10^{15} \mathrm{GeV}^{-1}$ and $1 \mathrm{~kg}=5.610 \times 10^{26} \mathrm{GeV}$ [34]

\section{Structure equations}

The model is described by the action

$$
S=S_{G}+S_{M}+S_{E M}
$$

where the gravity part $S_{G}$ is given by the usual EinsteinHilbert term, the electromagnetic Lagrangian $S_{E M}$ corresponds to the Maxwell theory, while the matter contribution corresponds to a perfect fluid with energy density $\rho$, pressure $p$ and a certain equation-of-state $F(p, \rho)=0$. Varying with respect to the metric tensor we obtain Einstein's field equations without a cosmological constant

$G_{\mu \nu}=R_{\mu \nu}-\frac{1}{2} R g_{\mu \nu}=8 \pi T_{\mu \nu}$

where we have set Newton's constant $G$ equal to unity. The total stress-energy tensor has two contributions

$T_{\mu \nu}=M_{\mu \nu}+E_{\mu \nu}$

namely one from the perfect fluid

$M_{\mu \nu}=p g_{\mu \nu}+(p+\rho) u_{\mu} u_{\nu}$

where $u_{\mu}$ is the four-velocity of the fluid, and one from the electromagnetic field [28]

$E_{\mu \nu}=\frac{1}{4 \pi}\left[-\frac{1}{4} F g_{\mu \nu}+F_{\nu \gamma} F_{\mu}^{\gamma}\right]$

where $F=F_{\mu \nu} F^{\mu \nu}$ is the Maxwell invariant, and $F_{\mu \nu}$ is the electromagnetic field strength. Furthermore, varying with respect to the Maxwell potential one obtains Maxwell's equations

$\nabla_{v} F^{\mu \nu}=4 \pi J^{\mu}$

where $J_{\mu}$ is the current of the charged fluid.

For the exterior problem, $r>R$, with $R$ being the radius of the star, where $M_{\mu \nu}$ vanishes, we seek static spherically symmetric solutions of the form

$\mathrm{d} s^{2}=-f(r) \mathrm{d} t^{2}+g(r) \mathrm{d} r^{2}+r^{2}\left(\mathrm{~d} \theta^{2}+\sin ^{2} \theta \mathrm{d} \phi^{2}\right)$

for the metric tensor, while for the electromagnetic field the only non-vanishing component is the one that corresponds to the electric field, $F_{t r}=E(r)=Q / r^{2}$. The solution to the exterior problem is of course the well-known ReissnerNordström solution [35]

$$
f(r)=g(r)^{-1}=1-\frac{2 M}{r}+\frac{Q^{2}}{r^{2}}
$$

where $M, Q$ are the mass and the electric charge, respectively, of the star. The electric charge takes values in the range $0 \leq Q \leq M$, where in the limit $Q \rightarrow 0$ we recover the Schwarzschild solution, while in the other limit the black hole becomes extremal.

For the interior solutions, $r<R$, we have to solve the field equations in the presence of both the perfect fluid and the electromagnetic field. As usual we make the ansatz

$\mathrm{d} s^{2}=-e^{\nu(r)} \mathrm{d} t^{2}+A(r) \mathrm{d} r^{2}+r^{2}\left(\mathrm{~d} \theta^{2}+\sin ^{2} \theta \mathrm{d} \phi^{2}\right)$

and we set for convenience

$A(r)^{-1}=1-\frac{2 m(r)}{r}+\frac{q(r)^{2}}{r^{2}}$

similar to the exterior solution, where $m(r)$ is the mass function, and $q(r)$ is the total electric charge function. Therefore, in total there are four unknown functions, $m(r)$, $q(r), v(r), p(r)$, satisfying the following system of coupled differential equations [28]

$$
\begin{aligned}
q^{\prime}(r)= & 4 \pi \rho_{e}(r) r^{2} \sqrt{A(r)} \\
m^{\prime}(r)= & 4 \pi \rho(r) r^{2}+\frac{q(r) q^{\prime}(r)}{r} \\
p^{\prime}(r)= & -(p(r)+\rho(r)) \frac{4 \pi r p(r)+\frac{m(r)}{r^{2}}-\frac{q(r)^{2}}{r^{3}}}{1-\frac{2 m(r)}{r}+\frac{q(r)^{2}}{r^{2}}} \\
& +\frac{q(r)}{r^{2}} \rho_{e}(r) \sqrt{A(r)} \\
v^{\prime}(r)= & \frac{1}{p(r)+\rho(r)}\left[\frac{q(r) q^{\prime}(r)}{2 \pi r^{4}}-2 p^{\prime}(r)\right]
\end{aligned}
$$

where $\rho_{e}(r)$ is the electric density, and the prime denotes differentiation with respect to the radial coordinate $r$. For neutral stars, $q(r)=0$, we recover the usual Tolman-OppenheimerVolkoff equations [36,37]. Finally, the differential equations are to be integrated imposing the initial conditions at the center of the star

$$
\begin{aligned}
p(0) & =p_{c} \\
m(0) & =0 \\
q(0) & =0
\end{aligned}
$$

with $p_{c}$ being the central pressure, while upon matching the solutions on the surface of the star, the following conditions must be satisfied 


$$
\begin{aligned}
p(R) & =0 \\
m(R) & =M \\
q(R) & =Q \\
e^{\nu(R)} & =1-\frac{2 M}{R}+\frac{Q^{2}}{R^{2}}
\end{aligned}
$$

The first condition allows us to determine the radius of the star, while the second and the third allows to compute both the mass and the charge of the object.

\section{Equation-of-state and numerical results}

\subsection{Equation-of-state}

To solve the structure equations we need to specify the sources first. Matter inside the star is modelled as a relativistic gas of de-confined quarks, and we consider an extension of the simplest MIT bag model $[38,39]$

$p(\rho)=\frac{1}{3}(\rho-4 B)$

with $B$ being the bag constant, where the EoS is linear in the form "radiation plus constant". In the present work we adopt a slightly more complicated, non-linear EoS obtained in the framework of color superconductivity [31-33], which is given by [40-42]

$$
\begin{aligned}
p(\rho) & =\frac{\rho}{3}-\frac{4 B}{3}+\frac{3 \gamma}{\pi^{2}} \mu^{2} \\
\mu^{2} & =-\gamma+\sqrt{\gamma^{2}+\frac{4}{9} \pi^{2}(\rho-B)}
\end{aligned}
$$

where the parameter $\gamma$ is defined by [42]

$\gamma=\frac{2}{3} \Delta^{2}-\frac{1}{6} m_{s}^{2}$

with $m_{s}$ being the mass of the strange quark, and $\Delta$ the energy gap. Clearly, when $\gamma=0$ we recover the simplest version of the MIT bag model mentioned before.

Given the equation-of-state $p=p(\rho)$, the speed of sound may be computed by $c_{s}^{2}=d p / d \rho$, and it is found to be

$c_{s}^{2}=\frac{1}{3}+\frac{2 \gamma}{3\left(\gamma+\mu^{2}\right)}$

Clearly, when $\gamma \rightarrow 0$ we recover the constant value $c_{s}=$ $1 / \sqrt{3}$ corresponding to the linear EoS, but when $\gamma \neq 0$ there is a non negligible effect, which leads to a density dependent value rather different than $1 / \sqrt{3}$. The behaviour of the speed of sound for neutral objects may be seen in Fig. 2 of [42].

In the CFL scenario, since the energy gap $\Delta \neq 0$, the EoS is non-linear even if the $s$ quark is taken to be massless.
In this case all three quark flavours, $u, d, s$, have the same number densities, and the neutrality condition

$(+2 / 3) n_{u}+(-1 / 3) n_{d}+(-1 / 3) n_{s}=0$

is satisfied without the presence of electrons [33]. When, however, $s$ quark has a finite mass, its number density is different than $n_{d}, n_{u}$, and the object without electrons acquires a net electric charge. For the electric density, following previous works $[24,27,28]$, we assume it is proportional to the energy density

$\rho_{e}(r)=\alpha \rho(r)$

with some constant of proportionality $\alpha$ taking values in the range $0 \leq \alpha \leq 1$ [28]. Since strange quark stars at low masses resemble an incompressible fluid, $\rho \approx \rho_{0}, M \sim R^{3}$, we find it natural to consider here an electric density proportional to matter energy density similar to [28], although different choices have been considered in other similar works, for instance a Gaussian profile [29] or a power-law for the total electric charge function [30]. It should be noted that although in principle the electric fraction $\alpha$ should depend on $m_{s}$, in the numerical analysis below we shall treat $\alpha$ as a free parameter, and we shall study its impact on properties of charged strange quark stars.

We remark in passing that other refine and more sophisticated quark EoSs do exist in the literature, such as models that incorporate a chiral symmetry breaking $[43,44]$ or models based on perturbative QCD studies [45,46], and others $[47,48]$. All these EoSs lead to stars with properties that vary in stiffness and compactness. In the following, however, we shall consider the EoS given above since it is always advantageous to work with analytic functions. It certainly would be interesting to study precisely how other more refine EoSs would affect the results of the present work, and we hope to be able to address that issue in a future work.

On the mass-radius plane the highest star mass that a given EoS can support crucially depends on the equation-of-state, and soft equations of state predict lower highest masses [49]. The observed massive pulsars PSR J1614-2230 with a mass (1.908 \pm 0.016$) M_{\odot}[50]$ and PSR J0348-0432 with a mass $(2.01 \pm 0.04) M_{\odot}[51]$ have put a stringent constrain on the EoS. Several EoSs predict maximum star masses well below $2 M_{\odot}$, and therefore they must be ruled out.

In the context of color superconductivity the EoS is characterized by three parameters, namely: $B, m_{s}, \Delta$, and there are 19 viable models, out of which 8 models admit a highest star mass larger than 2 solar masses, see e.g. [42]. In the following, we choose two fiducial models (number 10 and number 8) of [42] for our study, where the parameters take the following numerical values 



Fig. 1 First row electric charge $q(r)$ (left panel), pressure $p(r)$ (mid-

$\beta=M / R$ (middle panel) versus mass of the star (in solar masses), and dle panel) and metric function $e^{\lambda}$ (right panel) versus normalized radial mass-to-radius profiles (right panel) for $\alpha=0,0.1,0.2,0.4,0.4$ for the coordinate $r / R$ for $\alpha=0,0.1,0.2,0.4,0.4$ from bottom to top for the model CFL 10. Second row ratio $Q / M$ (left panel) and compactness
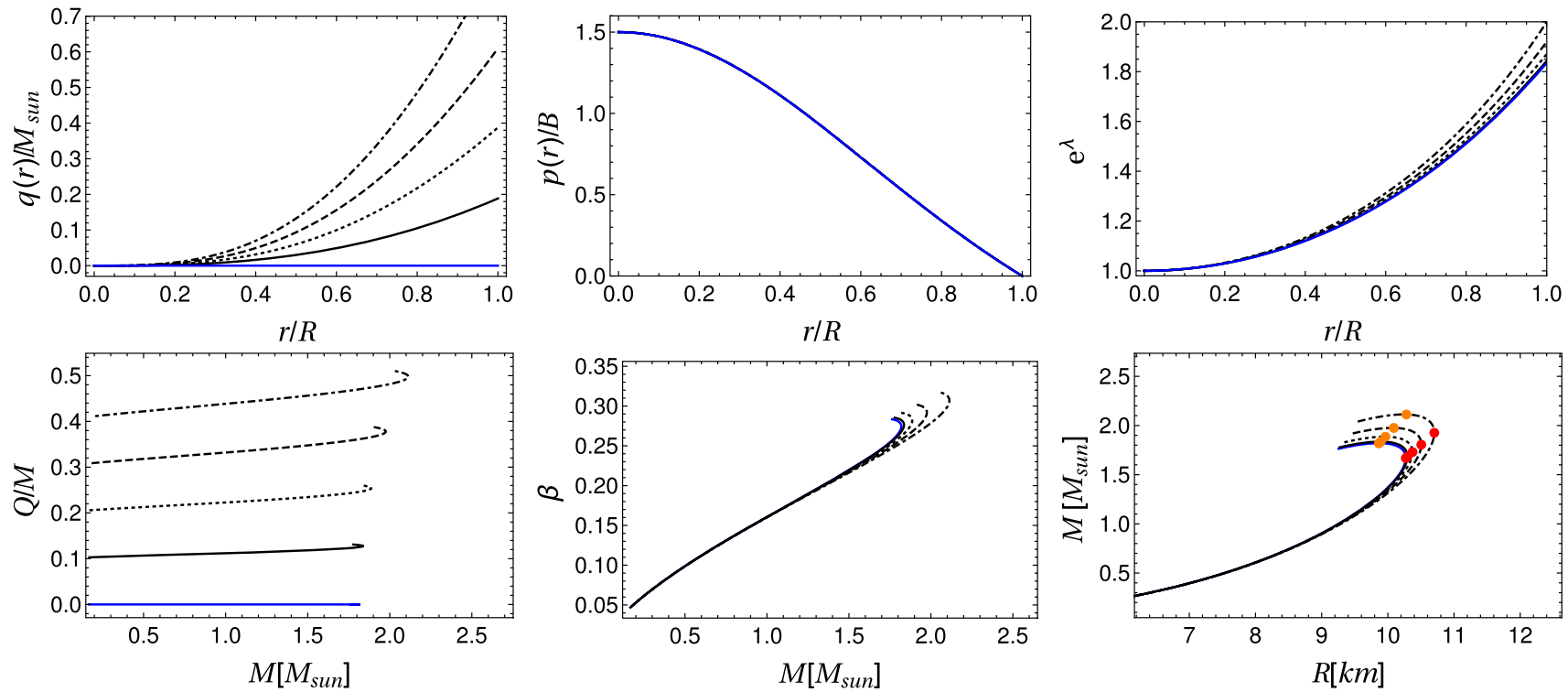

Fig. 2 Same as Fig. 1 but for the model CFL 8

Table 1 Maximum values of star mass (in solar masses) and radius (in $\mathrm{km}$ ) for the model CFL 10

Table 2 Maximum values of star mass (in solar masses) and radius (in $\mathrm{km}$ ) for the model CFL 8

\begin{tabular}{llllll}
\hline & $\alpha=0$ & $\alpha=0.1$ & $\alpha=0.2$ & $\alpha=0.3$ & $\alpha=0.4$ \\
\hline$R_{\max }$ & 11.87 & 11.90 & 11.98 & 12.12 & 12.34 \\
$M_{\max }$ & 2.20 & 2.22 & 2.28 & 2.38 & 2.55 \\
\hline & & & & \\
\hline & $\alpha=0$ & $\alpha=0.1$ & $\alpha=0.2$ & $\alpha=0.3$ & $\alpha=0.4$ \\
\hline$R_{\max }$ & 10.27 & 10.30 & 10.37 & 10.51 & 10.70 \\
$M_{\max }$ & 1.82 & 1.84 & 1.89 & 1.98 & 2.11 \\
\hline
\end{tabular}




$$
\begin{aligned}
\Delta & =150 \mathrm{MeV} \\
m_{s} & =150 \mathrm{MeV} \\
B & =80 \mathrm{MeV} \mathrm{fm}^{-3}
\end{aligned}
$$

and $M_{\max }=2.20 M_{\odot}$ for the model CFL 10 (above the two solar masses band) and

$$
\begin{aligned}
\Delta & =100 \mathrm{MeV} \\
m_{s} & =150 \mathrm{MeV} \\
B & =80 \mathrm{MeV} \mathrm{fm}^{-3}
\end{aligned}
$$

and $M_{\max }=1.82 M_{\odot}$ for the model CFL 8 (below the two solar masses band).

\subsection{Numerical results}

Our numerical results are summarized in the Figs. 1 and 2 for the models CFL10 and CFL8, respectively. First, in the top row of Fig. 1 we show the impact of the $\alpha$ parameter on the interior solutions for a given central pressure $p_{c}=1.5 \mathrm{~B}$. In particular, we show the charge function $q(r)$ (left panel), the pressure (middle panel) and the second metric function $e^{\lambda}$ (right panel) versus the normalized radial coordinate $r / R$ for $\alpha=0,0.1,0.2,0.3,0.4$ from bottom to top. Notice than in the case of the pressure all four curves coincide, since the pressure starts from the same central value and decreases until it vanishes at the surface of the star. The numerical results show that both the mass $M=m(R)$ and the electric charge $Q=q(R)$ increase with the $\alpha$ parameter.

In the bottom row of the same figure we show the massto-radius profile (right panel), the compactness $\beta=M / R$ versus mass (in solar masses, middle panel) as well as the electric charge versus mass of the star (left panel) for $\alpha=0,0.1,0.2,0.3,0.4$. We can see that the impact of the $\alpha$ parameter is significant only at higher masses, $1.5-2$ solar masses, while for light stars we do not see any observable effect. The numerical results show that the maximum radius, the maximum mass and the maximum compactness increase with the $\alpha$ parameter. Furthermore, for a given $\alpha$ the ratio $Q / M$ increases only slightly with the mass, while for a given mass it increases with $\alpha$. Finally, it is worth mentioning that a mass as high as 2 solar masses can be supported, and that the charged stars are heavier than their neutral counterparts. Regarding the mass-toradius profiles, the highest values $M_{\max }, R_{\max }$ are shown in Table 1.

Next, we show in the top row of Fig. 2 the impact of the $\alpha$ parameter on the interior solutions for a given central pressure $p_{c}=1.5 \mathrm{~B}$ for the model CFL8. Similarly to the previous model, first we show the charge function $q(r)$ (left panel), the pressure (middle panel) and the second metric function $e^{\lambda}$ (right panel) versus the normalized radial coordinate $r / R$ for $\alpha=0,0.1,0.2,0.3,0.4$ from bottom to top. We then observe a similar behaviour to the model CFL10. In this case when we decrease the value of $\Delta$, the first and the third plots are attenuated, whereas the pressure remains intact. The mass-toradius profiles obtained here are similar to the ones obtained in $[29,30]$.

In the bottom row of the same figure, we show the mass-toradius profile (right panel), the compactness $\beta=M / R$ versus mass (in solar masses, middle panel) as well as the electric charge $Q / M$ versus mass of the star (left panel) for the same values of $\alpha$ considered in the previous model. Regarding the mass-to-radius profiles, the highest values $M_{\max }, R_{\max }$ are shown in Table 2. In comparison with the model CFL10 we obtain qualitatively similar results (although the stars of the model CFL8 are somewhat smaller and lighter than the ones of the model CFL10), namely (i) both the mass $M$ and the electric charge $Q$ increase with the $\alpha$ parameter, (ii) the charged stars are heavier than their neutral counterparts, and (iii) the compactness is lower than 0.35 , which is compatible with the Buchdahl bound, $\beta \leq 4 / 9 \simeq 0.44$ [52]. Notice that although in the case of neutral stars in the CFL 8 model the highest mass supported by the assumed EoS does not cross the two solar masses band, in the case of charged stars, and when the electric charge becomes sufficiently large, the highest mass eventually reaches the two solar masses bound. Furthermore, the event GW170817 [53] has imposed a new constraint to the upper limit of the maximum mass, $2.17 M_{\odot}$ [54], and consequently the model CFL 10 would be ruled out.

\section{Conclusions}

In summary, in the present work we have studied the properties of electrically charged strange quark stars. We have assumed a non-linear equation-of-state which describes quark matter in the framework of color superconductivity, and we have obtained numerical solutions to the structure equations. The impact of the charge density on properties of the stars is investigated in detail. Our results show that (i) electric stars are heavier than their neutral counterparts, (ii) the predicted mass-to-radius profiles cross the 2 solar mass band, (iii) both the mass and the charge of the stars increase with the $\alpha$ parameter of the charge density for a given central pressure, and (iv) the maximum radius, the maximum mass and the maximum compactness increase with the $\alpha$ parameter.

Acknowledgements We wish to thank the anonymous reviewer for useful comments and suggestions. The author G. P. thanks the Fundação para a Ciência e Tecnologia (FCT), Portugal, for the financial support to the Center for Astrophysics and Gravitation-CENTRA, Instituto Superior Técnico, Universidade de Lisboa, through the Grant No. 
UID/FIS/00099/2013. The author A. R. acknowledges DI-VRIEA for financial support through Proyecto Postdoctorado 2019 VRIEA-PUCV.

Data Availability Statement This manuscript has no associated data or the data will not be deposited. [Authors' comment: It is a theoretical work and therefore no data were used.]

Open Access This article is distributed under the terms of the Creative Commons Attribution 4.0 International License (http://creativecomm ons.org/licenses/by/4.0/), which permits unrestricted use, distribution, and reproduction in any medium, provided you give appropriate credit to the original author(s) and the source, provide a link to the Creative Commons license, and indicate if changes were made.

Funded by SCOAP ${ }^{3}$.

\section{References}

1. S.L. Shapiro, S.A. Teukolsky, Black Holes, White Dwarfs, and Neutron Stars: The Physics of Compact Objects (Wiley, New York, 1983), p. 645

2. D. Psaltis, Living Rev. Rel. 11, 9 (2008). arXiv:0806.1531 [astro$\mathrm{ph}]$

3. D.R. Lorimer, Living Rev. Rel. 11, 8 (2008). arXiv:0811.0762 [astro-ph]

4. A. Einstein, Ann. Phys. 49, 769822 (1916)

5. W. Baade, F. Zwicky, Phys. Rev. 45, 130 (1934)

6. J. Chadwick, Nature 129, 312 (1932)

7. J. Chadwick, Proc. R. Soc. Lond. A 136(830), 692 (1932)

8. N. Chamel, Phys. Rev. Lett. 110(1), 011101 (2013). arXiv:1210.8177 [astro-ph.HE]

9. A. Hewish, S.J. Bell, J.D.H. Pilkington, P.F. Scott, R.A. Collins, Nature 217, 709 (1968)

10. E. Witten, Phys. Rev. D 30, 272 (1984)

11. E. Farhi, R.L. Jaffe, Phys. Rev. D 30, 2379 (1984)

12. E.O. Ofek et al., Astrophys. J. 659, L13 (2007). arXiv:astro-ph/0612408

13. R. Ouyed, D. Leahy, P. Jaikumar, arXiv:0911.5424 [astro-ph.HE]

14. C. Alcock, E. Farhi, A. Olinto, Astrophys. J. 310, 261 (1986)

15. C. Alcock, A. Olinto, Ann. Rev. Nucl. Part. Sci. 38, 161 (1988)

16. J. Madsen, Lect. Notes Phys. 516, 162 (1999)

17. F. Weber, Prog. Part. Nucl. Phys. 54, 193 (2005). arXiv:astro-ph/0407155

18. Y.L. Yue, X.H. Cui, R.X. Xu, Astrophys. J. 649, L95 (2006). arXiv:astro-ph/0603468

19. D. Leahy, R. Ouyed, Mon. Not. Roy. Astron. Soc. 387, 1193 (2008). arXiv:0708.1787 [astro-ph]

20. K. Schwarzschild, Sitzungsber. Preuss. Akad. Wiss. Berlin (Math. Phys.) 1916, 189 (1916). arXiv:physics/9905030

21. J.D. Bekenstein, Phys. Rev. D 4, 2185 (1971)

22. F. de Felice, Y.-Q. Yu, J. Fang, Mon. Not. R. Astron. Soc. 277, L17 (1995)

23. F. de Felice, S m Liu, Y q Yu, Class. Quant. Gravit. 16, 2669 (1999). arXiv:gr-qc/9905099
24. J.L. Zhang, W.Y. Chau, T.Y. Deng, Astrophys. Space Sci. 88, 81 (1982)

25. S. Ray, A.L. Espindola, M. Malheiro, J.P.S. Lemos, V.T. Zanchin, Phys. Rev. D 68, 084004 (2003). arXiv:astro-ph/0307262

26. B.B. Siffert, J.R. de Mello, M.O. Calvão, Br. J. Phys. 37, 609 (2007)

27. J.D.V. Arbail, J.P.S. Lemos, V.T. Zanchin, Phys. Rev. D 88, 084023 (2013). arXiv:1309.4470 [gr-qc]

28. J.D.V. Arbail, J.P.S. Lemos, V.T. Zanchin, Phys. Rev. D 89(10), 104054 (2014). arXiv:1404.7177 [gr-qc]

29. R.P. Negreiros, F. Weber, M. Malheiro, V. Usov, Phys. Rev. D 80, 083006 (2009). arXiv:0907.5537 [astro-ph.SR]

30. J.D.V. Arbail, M. Malheiro, Phys. Rev. D 92, 084009 (2015). arXiv:1509.07692 [astro-ph.SR]

31. M.G. Alford, K. Rajagopal, F. Wilczek, Nucl. Phys. B 537, 443 (1999). arXiv:hep-ph/9804403

32. K. Rajagopal, F. Wilczek, Phys. Rev. Lett. 86, 3492 (2001). arXiv:hep-ph/0012039

33. M. Alford, K. Rajagopal, JHEP 0206, 031 (2002). arXiv:hep-ph/0204001

34. A.H. Guth, Phys. Rev. D 23, 347 (1981)

35. H. Reissner, Ann. Phys. 355, 106-120 (1916)

36. J.R. Oppenheimer, G.M. Volkoff, Phys. Rev. 55, 374 (1939)

37. R.C. Tolman, Phys. Rev. 55, 364-73 (1939)

38. A. Chodos, R.L. Jaffe, K. Johnson, C.B. Thorn, V.F. Weisskopf, Phys. Rev. D 9, 3471 (1974)

39. A. Chodos, R.L. Jaffe, K. Johnson, C.B. Thorn, Phys. Rev. D 10, 2599 (1974)

40. G. Lugones, J.E. Horvath, Phys. Rev. D 66, 074017 (2002). arXiv:hep-ph/0211070

41. C Vasquez Flores, G. Lugones, Phys. Rev. D 82, 063006 (2010). arXiv: 1008.4882 [astro-ph.HE]

42. C.V. Flores, G. Lugones, Phys. Rev. C 95(2), 025808 (2017). arXiv: 1702.02081 [astro-ph.HE]

43. Y. Nambu, G. Jona-Lasinio, Phys. Rev. 122, 345 (1961)

44. Y. Nambu, G. Jona-Lasinio, Phys. Rev. 124, 246 (1961)

45. E.S. Fraga, A. Kurkela, A. Vuorinen, Astrophys. J. 781(2), L25 (2014). arXiv:1311.5154 [nucl-th]

46. A. Vuorinen, arXiv:1611.04557 [hep-ph]

47. V.D. Toneev, E.G. Nikonov, B. Friman, W. Norenberg, K. Redlich, Eur. Phys. J. C 32, 399 (2003). arXiv:hep-ph/0308088

48. Y.B. Ivanov, A.S. Khvorostukhin, E.E. Kolomeitsev, V.V. Skokov, V.D. Toneev, D.N. Voskresensky, Phys. Rev. C 72, 025804 (2005). arXiv:astro-ph/0501254

49. S. Weissenborn, D. Chatterjee, J. Schaffner-Bielich, Nucl. Phys. A 881, 62 (2012). arXiv:1111.6049 [astro-ph.HE]

50. Z. Arzoumanian et al. [NANOGrav Collaboration], Astrophys. J. Suppl. 235(2), 37 (2018). arXiv:1801.01837 [astro-ph.HE]

51. J. Antoniadis et al., Science 340, 6131 (2013). arXiv:1304.6875 [astro-ph.HE]

52. H.A. Buchdahl, Phys. Rev. 116, 1027 (1959)

53. B.P. Abbott et al. [LIGO Scientific and Virgo Collaborations], Phys. Rev. Lett. 119(16), 161101 (2017). arXiv:1710.05832 [gr-qc]

54. B. Margalit, B.D. Metzger, Astrophys. J. 850(2), L19 (2017). arXiv:1710.05938 [astro-ph.HE] 